\title{
THE EARLY DIASTOLIC SOUND OF CONSTRICTIVE PERICARDITIS
}

\author{
BY \\ PATRICK MOUNSEY \\ From the Cardiac Department of the London Hospital \\ Received September 9, 1954
}

Evans and Jackson (1952), in 27 out of 30 cases of constrictive pericarditis, found an adventitious sound in early diastole which they ascribed in some to splitting of the second sound and in others to a third heart sound. Further research, however, has suggested that this added sound is probably a sound peculiar to constrictive pericarditis (Evans, 1954).

Potain (1856) described an added sound in early diastole in constrictive pericarditis in these words :

" The second sound was reduplicated or rather composed of two sounds, following one another in quick succession; one, clear, dry, ringing, with the usual characteristics of a valvular snap and of more or less unchanging intensity; the other coming immediately afterwards, less well defined, less resonant, coinciding exactly with the impulse felt in the præcordial region, like the impulse increasing during inspiration, and taking on at that point a rather metallic tone."

Lian et al. (1933) named the sound the pericardial protodiastolic vibration. Eliasch et al. (1950) showed that the sound coincided with the nadir of the early diastolic dip, recorded in right heart pressure records in constrictive pericarditis. More recently McKusick (1952), with simultaneous records of heart sound and ventricular border electrokymograms demonstrated that the sound coincided with an abrupt halt in rapid ventricular filling in early diastole. The purpose of the present study was to investigate further the clinical significance and pathogenesis of this sound.

\section{METHOD}

In 22 patients with constrictive pericarditis the heart sounds were examined clinically and in the phonocardiogram, and all patients were subsequently treated by operation. There were 19 men and 3 women in the series, with an average age of 36 years. Auricular fibrillation was present in eight cases. Phonocardiograms were recorded on a four-channel phonocardiograph using crystal microphones, as described by Leatham (1949). In 15 patients pre- and post-operative phonocardiograms were obtained. Right heart catheterization was performed before operation in six patients, using a capacitance electromanometer, critically damped, and a two-channel direct recorder. Simultaneous phonocardiograms and pressure records were obtained in five patients, the two records being made on separate instruments and synchronized by an electrocardiogram, which was used as a common reference tracing. Control experiments showed that no significant error in timing was introduced by the synchronous use of these two separate instruments.

\section{RESULTS}

An early diastolic sound was present before operation in 18 out of the 22 patients (Fig. 1). It was best heard over the centre of the præcordium and varied considerably in intensity. When loud it often had a snapping quality; it might be louder even than the first and second heart sounds. It 




FIG. 1.-Phonocardiogram of the early diastolic sound, before operation. The early diastolic sound (EDS) is of comparable intensity to the first heart sound (1) and of greater intensity than the second sound (2) The interval between the beginning of the second sound and the peak of the deflection of greatest amplitude in the early diastolic sound is $0.1 \mathrm{sec}$. $L S E=$ Left sternal edge. $L F=$ low frequency. $C R_{1}=$ Electrocardiographic lead $C R_{1}$.

was often accompanied by a palpable diastolic thrust, as described by Potain (1856) and Wood (1950), and on a simultaneous phonocardiogram and apex cardiogram the sound was seen to coincide with this thrust. On inspiration the sound usually increased in intensity. In measuring the place of the sound in the cardiac cycle, the peak or nadir of the single vibration of greatest amplitude and/or highest frequency in the early diastolic sound was used, as a convenient point to measure. The place of the sound appeared to be the same as that of the opening snap of mitral stenosis, but the phonocardiogram in 15 patients before operation showed that the interval between it and the beginning of the second sound lay between 0.09 and $0.13 \mathrm{sec}$., the average being $0.1 \mathrm{sec}$., whereas the range for the opening snap was between 0.03 and $0.12 \mathrm{sec}$., with an average of $0.07 \mathrm{sec}$. (Mounsey, 1953).

After a successful operation, the sound became softer and was delayed so that it resembled the third heart sound both in its character and its place in the cardiac cycle (Fig. 2). The lessened intensity of the early diastolic sound after operation appeared to be related to the fall in jugular venous pressure. In the four patients in whom the sound remained loud, the pressure remained

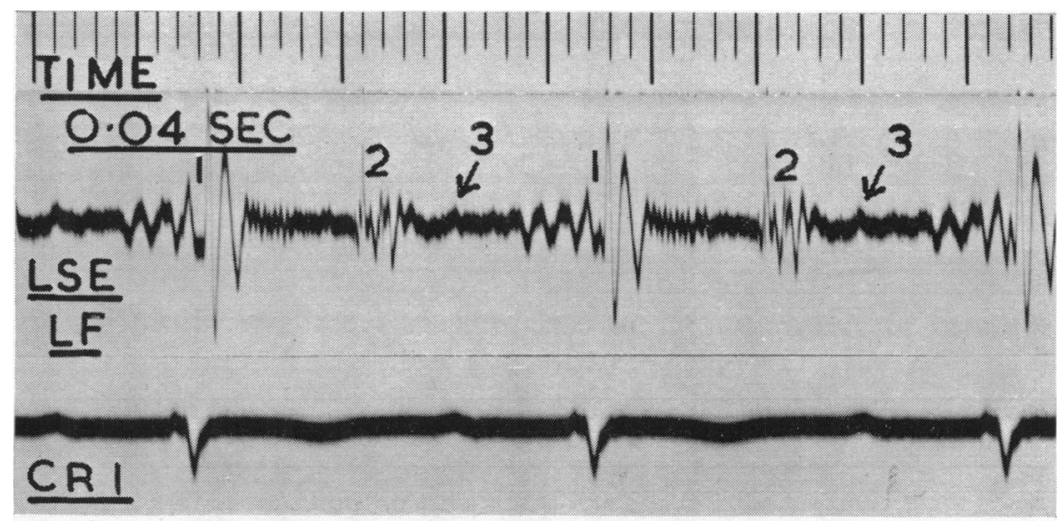

FIG. 2.-Phonocardiogram in the same patient as in Fig 1, after successful operation. The early diastolic sound has been replaced by a small third heart sound (3). The interval between the beginning of the second sound (2) and the peak of the third heart sound is $0 \cdot 18 \mathrm{sec}$. 
markedly raised, whereas in the two patients in whom the pressure fell to a normal level after operation, the sound was very soft. The interval between the beginning of the second sound and the early diastolic sound increased in every patient after operation and lay between 0.14 and $0.2 \mathrm{sec}$., with an average of $0.17 \mathrm{sec}$. It is unlikely that this marked change could be accounted for by the slightly reduced heart rate in six of the patients at the time of the postoperative phonocardiogram, since variations in rate appeared to have little effect on the timing of the sound in repeated phonocardiograms on the same patients. No direct correlation was noted between the amount of delay of the early diastolic sound postoperatively and the degree of fall of jugular venous pressure.

No clear early diastolic sound was heard before operation in four patients. They appeared comparable with the remaining 18 patients in regard to the height of the jugular venous pressure and the degree of liver enlargement. It was noted, however, in three of these four that the movement of the heart at operation differed from that seen in patients in whom an early diastolic sound was present. In patients having the sound, abrupt halting of ventricular expansion was seen in early diastole, while in the three patients without an added sound, ventricular dilatation in early diastole appeared less sudden and no abrupt halting was seen.

No direct correlation was noted between the presence of calcification of the pericardium and the early diastolic sound. Calcification was present in two of the four cases not having the sound and was very extensive in one, and absent in the other two. It was present in 11 of the 18 cases having the sound and absent or slight in the remaining seven.

The early diastolic sound is a useful clinical sign of constrictive pericarditis. It can be differentiated from a split second sound by its character, being less well defined and ringing, by its wide area of propagation, being usually widely heard over the whole præcordium, and by its timing, the interval between it and the beginning of the second sound being greater than that between the two components of a split second sound. A further point in recognition is that a split second sound is nearly always present in addition to the early diastolic sound, although admittedly often difficult to tell clinically. It differs from a third heart sound in that it is usually louder, more clear-cut, is heard over a wide area of the præcordium and is nearer in time to the beginning of the second heart sound than is the third sound. The increase on inspiration in intensity of the sound and in the coincidental diastolic thrust is a further guide to the recognition of the early diastolic sound.

The early diastolic sound is particularly useful in cases where pericardial calcification is not seen on X-ray examination, since here its presence may help to decide between heart failure and constrictive pericarditis. Infrequently the sound is absent in constrictive pericarditis so that this should not exclude the diagnosis.

The definite relationship between the lessening of the intensity of the sound after operation and the degree of fall of jugular venous pressure explains why this change in the sound predicts a favourable postoperative progress, as noted by Evans and Jackson (1952). In some patients, however, in spite of clinical improvement after operation, the sound remained loud, but in these the venous pressure did not return to a normal level.

Pathogenesis of the Sound. It was considered that this added sound in constrictive pericarditis is caused by an abrupt halting of rapid ventricular filling by the rigid constricting pericardium. In order to test this theory, simultaneous phonocardiograms and right heart pressure measurements, and pressure differences between the right heart and peripheral veins were examined.

In pressure records from the right ventricle and the right atrium, the early diastolic dip characteristic of constrictive pericarditis (Bloomfield et al., 1946; Hansen et al., 1951; and McKusick, 1952), was seen in the six patients examined by cardiac catheterization. In four both the right atrium and the right ventricle were entered, while in two, records were only obtained from the right atrium. The nadir of the early diastolic dip was approximately 0.01 to 0.02 second later in the atrium than in the ventricle. The early diastolic sound coincided closely with the beginning of the steep upstroke of the early diastolic dip in all four patients having the sound and in whom simultaneous sound and pressure records were taken (Fig. 3). 


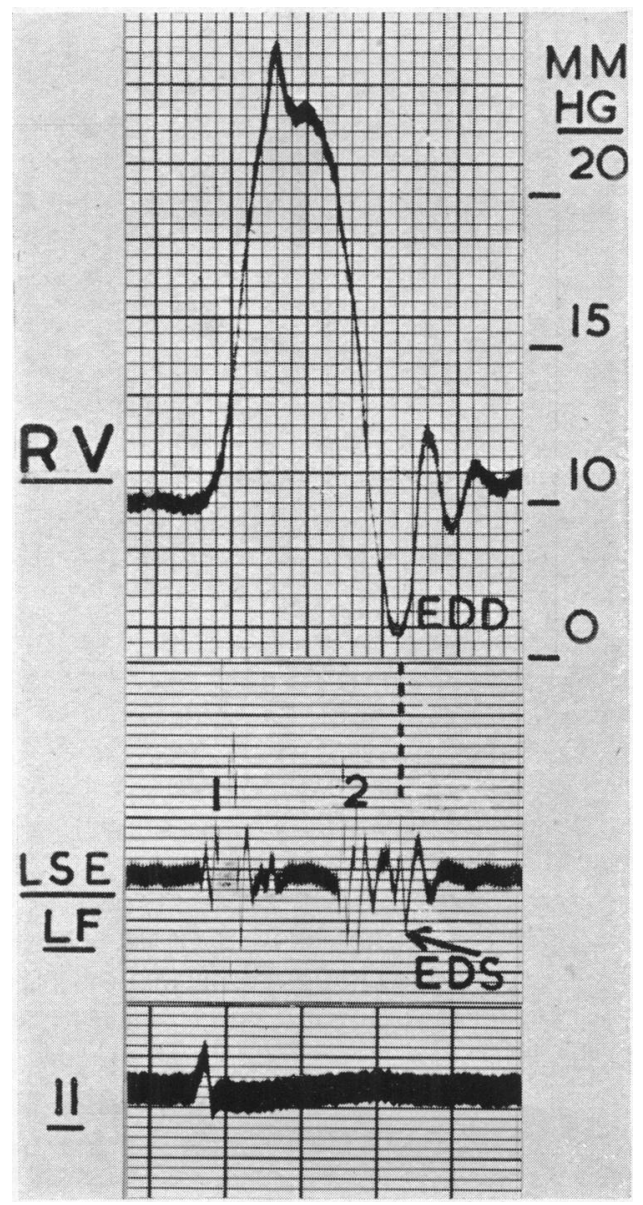

FIG. 3.-Simultaneous phonocardiogram and right ventricular pressure record. The early diastolic sound (EDS) coincides with the beginning of the steep upstroke of the early diastolic dip (EDD). RV=Right ventricular pressure. LSE LF $=$ Phonocardiogram at left sternal edge with low frequency filter.

The pressure difference between the peripheral veins and right heart during the early diastolic dip was then examined in order to try and estimate variations in blood flow in early diastole. Pressure recordings from the superior vena cava, the left innominate vein, the subclavian vein, and the basilic vein were made in four patients, on withdrawal of the catheter from the right heart. Relative pressure during the cardiac cycle at this series of positions were reconstructed by the use of a synchronous electrocardiogram (Fig. 4). In this way it was shown that there existed during the first portion of the early diastolic dip, a rapidly increasing pressure difference, of graduated degree, at points between the basilic vein and the right ventricle. At the beginning of the steep upstroke of the early diastolic dip, when the sound occurred, this process was reversed and the pressure difference diminished rapidly. There was usually a small time lag of varying degree in the pressure dip in the distal veins compared with that in the right heart, as indicated by the slope of the dotted line in Fig. 4 and 5. A similar pressure difference was shown to exist between the inferior vena cava and the right atrium and ventricle, although in the case of the inferior vena cava there was an abrupt, as opposed to a gradual change, in the form of the wave on passing from the inferior vena cava to the right atrium.

These pressure differences between the right heart and the basilic vein suggest that, during the descending limb of the early diastolic dip, rapid ventricular filling is taking place, since the pressure difference is then increasing steeply, and that during the upstroke of the dip, filling rapidly decreases, 


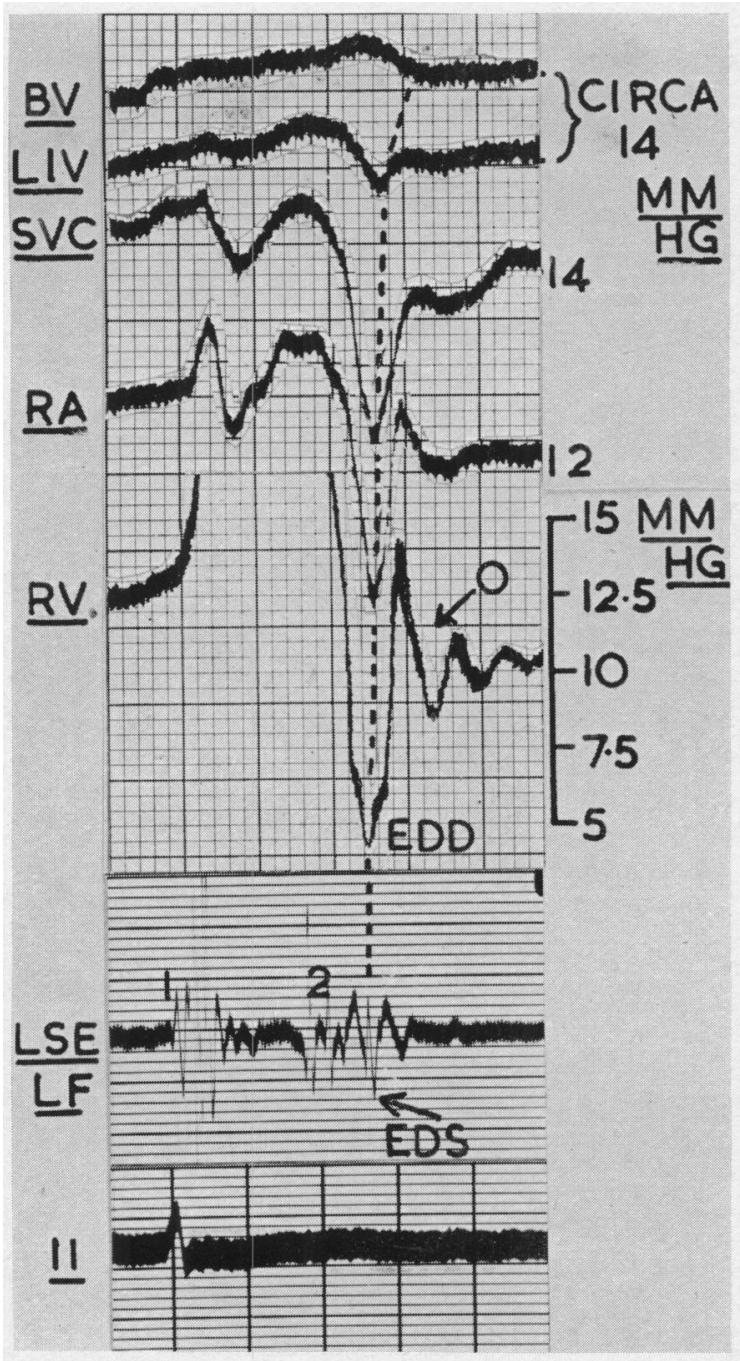

FIG. 4.-Synchronized pressure tracings from right ventricle (RV), right atrium (RA), superior vena cava (SVC), left innominate vein (LIV) and basilic vein (BV). The phonocardiogram (LSE.LF) was recorded simultaneously with the right ventricular pressure record and shows the position of the early diastolic sound (EDS).

The values for right ventricular pressures are shown on the right of the record. The scale for the other pressure tracings is the same, but, because of lack of space, only a single value is shown for each tracing at the point where it intersects the right-hand edge of the record.

The early diastolic dip (EDD) decreases in depth at greater distances from the heart until in the basilic vein no dip is recorded. Similarly the nadir of the early diastolic dip becomes slightly later in time, at greater distances from the heart, as indicated by the slope of the dotted line. Note the coarse oscillations (O) following the steep upstroke of the dip.

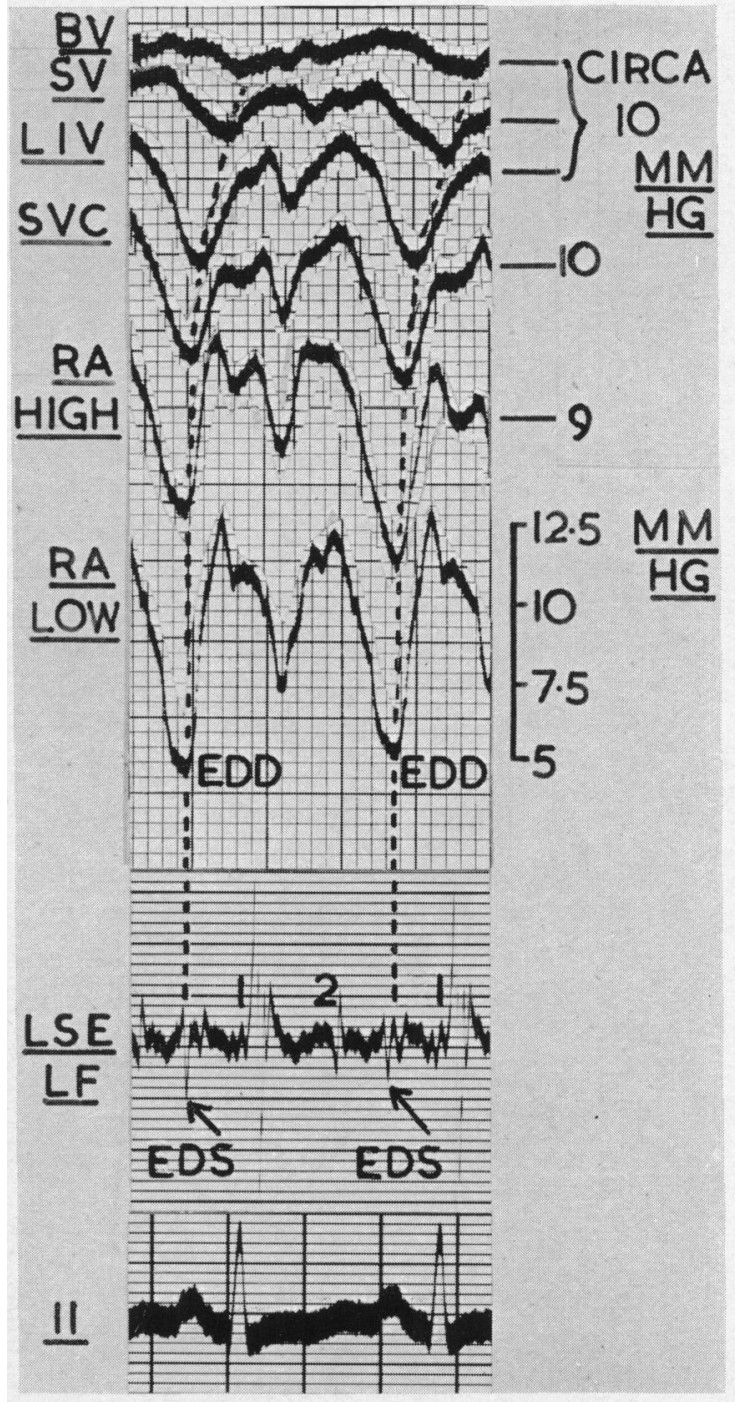

FIG. 5.-Synchronized pressure tracings from right atrium (RA) at points low and high within the atrium, superior vena cava (SVC), left innominate vein (LIV), subclavian vein (SV), and basilic vein (BV).

This graph was constructed in the same way as Fig. 4 and illustrates the same points, but with the difference that the delay in timing of the nadir of the early diastolic dip (EDD), at greater distances from the heart, is more marked in this patient than in the patient in Fig. 4. The delay is indicated by the slope of the dotted line. 
since the pressure difference is falling abruptly. The abruptness of the halting of rapid filling is suggested by the very steep rise of pressure in the right ventricle and the coarse oscillations following it. The fact that the beginning of the rise in pressure in the right ventricle precedes that in the right atrium, superior vena cava, and innominate vein suggests that the sudden cessation in rapid filling originates in the ventricle.

These observations, therefore, indicate that the early diastolic sound is synchronous with the beginning of the steep upstroke of the early diastolic dip, and thus colncides with abrupt halting of rapid ventricular filling.

Collateral evidence that abrupt halting of rapid ventricular filling is the mechanism responsible for the early diastolic sound and the steep upstroke of the early diastolic dip was obtained from an experiment with a simple model, which is described in the appendix to this paper.

Absence of the Early Diastolic Sound. Absence of the sound in some patients was considered to be due to a slower ventricular filling and a less abrupt halting, an opinion supported by observations of the heart movements at thoracotomy. The difference noted in the form of the pressure records from the right heart in patients having the sound, as opposed to those without it, also supported this theory. In the four patients having the sound, the upstroke of the dip in right ventricular and atrial pressure curves was more abrupt and was followed in two of these by coarse oscillations. In the two patients not having the sound, on the other hand, the upstroke of the early diastolic dip was less abrupt and merged smoothly into a diastolic plateau, which was undisturbed by the coarse oscillations seen in the group of patients having the sound (Fig. 6).

\section{DisCUSSION}

The evidence advanced in this study supports the concept of Hansen et al. (1951) and McKusick (1952) that the early diastolic sound in constrictive pericarditis is the result of abrupt halting of rapid ventricular filling by the rigid pericardium and is probably a waterhammer phenomenon. The exact mechanism of production of the sound, however, remains in doubt. Lian et al. (1933) suggested that the sound emanated from the vibrations of the calcified pericardium itself, when abruptly distended. Wood (1950) thought that it might be caused by the rapidly filling heart meeting the unyielding resistance of the pericardium which from a state of relaxation is thrown abruptly into tension. Eliasch et al. (1950) suggested that it resulted from turbulence in the right ventricle due to rapid filling.

Simultaneous phonocardiograms and right heart pressure records in this investigation have confirmed the finding of Eliasch et al. (1950), that the early diastolic sound coincided with the beginning of the steep upstroke of the early diastolic dip. In addition, however, pressure differences between the right heart, superior vena cava, left innominate vein, and basilic vein during the early diastolic dip have been measured in this study and these have added further evidence that the early diastolic sound coincides with abrupt halting of rapid right ventricular filling.

The early diastolic dip has been accepted as a real phenomenon in this investigation, although its nature and cause are still debatable. Wood et al. (1948) considered that it might be a distortion produced by their low frequency recording system. McKusick (1952) thought that the dip was probably a real phenomenon and that, while its pathogenesis was not fully understood, a ventricular sucking action, as a result of elastic recoil of the adherent pericardial casing, could not be excluded. Bloomfield et al. (1946) and Hansen et al. (1951), on the other hand, pointed out that the dip is also seen in cases of right heart failure due to any cause and in these cases ventricular suction as a result of pericardial recoil cannot be invoked to explain the dip.

It seemed to us that the problem was not so much why the dip occurred, since an abrupt fall of right ventricular pressure to near intrathoracic levels is likely to occur in early diastole, if ventricular relaxation is sufficiently rapid, but rather why the pressure in the more peripheral veins, such as the basilic vein, did not fall simultaneously. One factor that might explain this is the fact that the cross-sectional area of the total venous bed diminishes as blood flows centrally, from the peripheral vessels to the right heart (Green, 1943) and hence blood, entering the superior vena cava is in fact 


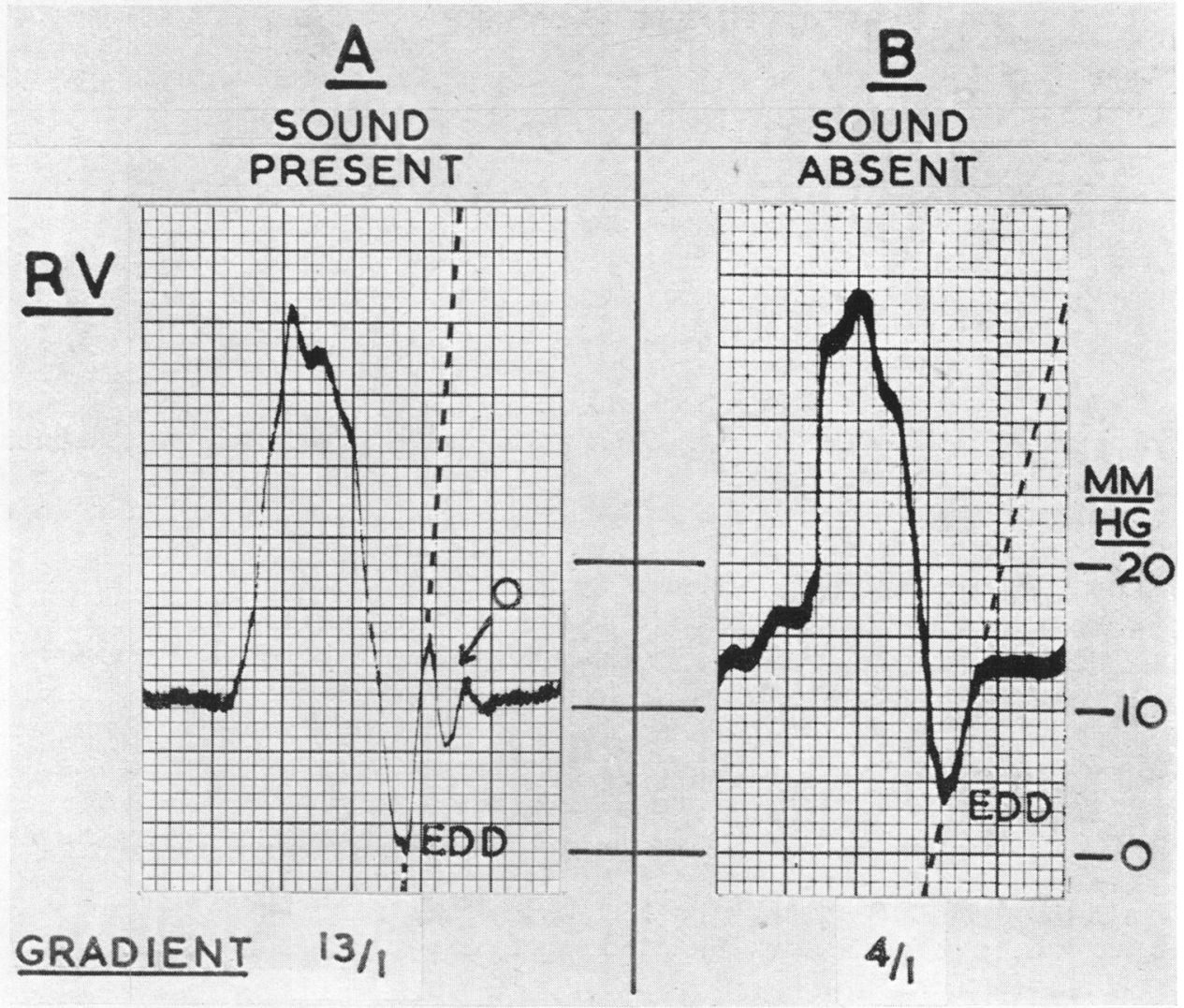

FIG. 6.-Comparison of the steepness of the upstroke of the early diastolic dip (EDD) in the right ventricle (RV), in patients with and without an early diastolic sound in constrictive pericarditis.

The dotted line indicates the gradient of the steepest part of the upstroke.

The steepness of the upstroke in patient A, having the sound (gradient 13/1), is contrasted with the gentler upstroke (gradient $4 / 1$ ) in patient $B$, not having the sound. In addition coarse oscillations (o) followed the upstroke in patient $\mathrm{A}$, having the sound, whereas these were absent in patient $\mathrm{B}$, not having the sound.

entering a relative constriction. For this reason relatively large variations in pressure and flow in the venæ cavæ are accompanied by relatively small variations in the more peripheral venous bed.

The rise in pressure at the end of the early diastolic dip is almost certainly due to filling of the ventricle to near capacity, with the result that the pressure in the ventricle rises to the level of that in the peripheral veins. The finding that this rise of pressure was less abrupt and not followed by coarse oscillations, in those patients with constrictive pericarditis who did not have the sound, supports the idea that suddenness of halting of filling is necessary for the production of the sound. This would also explain why, in patients with right heart failure due to other causes, in spite of the presence of an early diastolic pressure dip, the loud early diastolic sound of constrictive pericarditis is not heard. In such patients halting of filling is less abrupt and is cushioned by the distensible myocardium, thus giving rise to a relatively soft third heart sound.

After successful operation, the main factors, causing a change in the intensity and timing of the early diastolic sound, were thought to be the removal of the rigid constricting pericardial casing, the consequent increase in diastolic heart volume and the decrease in venous filling pressure. Blood flows less rapidly into the ventricles in early diastole, because of the decreased venous pressure, and hence the sound produced by filling arrest tends to become softer and delayed. The increased 
diastolic cardiac volume is a second factor that by prolonging ventricular filling tends to delay the sound. Lastly the partial removal of the rigid pericardial casing takes away to a large extent the mechanism responsible for sudden halting, which, as described above, is probably an essential factor in the production of the early diastolic sound. Thus, in cases where stripping has been extensive, the early diastolic sound, although it may have been prominent before operation, may become indistinguishable afterwards from a soft third heart sound.

It is possible and indeed probable that flow and pressure changes in the left heart also contribute to the genesis of the early diastolic sound. In this paper, however, in the absence of observations on the hæmodynamic events in the left atrium and ventricle, only the events in the right heart have been considered in relation to the genesis of the sound.

\section{SUMMARY AND CONCLUSIONS}

An added sound in early diastole was heard and recorded in the phonocardiogram in 18 out of 22 patients with constrictive pericarditis.

The early diastolic sound varied in intensity and character, but was often loud and snapping, and often accompanied by a palpable diastolic thrust. The average time interval between it and the beginning of the second sound was $0.1 \mathrm{sec}$.

After a successful operation of cardiac decompression, the sound always became softer and the average time interval between it and the second sound increased to $0 \cdot 17 \mathrm{sec}$.

Simultaneous sound and pressure records from the right heart were obtained and the early diastolic sound was found to coincide with the beginning of the steep upstroke of the early diastolic pressure dip, characteristic of constrictive pericarditis. Pressure differences were measured between the right heart, the superior vena cava, and left innominate vein during the early diastolic dip. These observations indicated that the sound coincided with the abrupt halting of rapid right ventricular filling.

Evidence from right heart pressure records and from direct inspection of the heart at thoracotomy suggested that slower ventricular filling followed by less abrupt halting accounted for absence of the sound in some patients.

The early diastolic sound is a useful clinical sign and its presence supports the diagnosis of constrictive pericarditis. Its occasional absence, however, should not exclude the diagnosis.

An experiment described in an appendix supports the view that the early diastolic sound is due to abrupt halting of ventricular filling.

\section{APPENDIX}

An Experiment. The early diastolic sound, the early diastolic pressure dip and the pressure difference between the right heart and peripheral veins were imitated in a simple experimental model, in which abrupt halting of rapid ventricular filling was simulated (Fig. 7).

Water was led from a large reservoir, representing the total peripheral venous bed, through a pipe, representing the superior vena cava, into a collapsible but non-distensible rubber tube, representing the ventricle enclosed in a constricting pericardium. The pressure head in the reservoir was $30 \mathrm{~mm}$. $\mathrm{Hg}$ and was constant throughout the experiment. The pipe was approximately $2 \mathrm{~cm}$. in diameter and $30 \mathrm{~cm}$. long. The capacity of the rubber tube, which could be closed at its distal end by a rubber membrane was 50 c.c. Pressure was measured in the rubber tube and reservoir by means of a cardiac catheter and simultaneous sound recordings were made with the microphone attached to the rubber membrane.

When no flow occurred in the system the pressure in the rubber tube was equal to that in the reservoir (Fig. 7A). When the rubber membrane was removed and steady flow occurred, the pressure in the tube approached atmospheric levels, while that in the reservoir remained unchanged (Fig. 7B). When a clamp was released from the end of the tube and the water accelerated from rest before reaching steady flow, a rapid fall in pressure in the tube took place, the pressure finally settling at the level previously recorded during steady flow (Fig. 7C). 


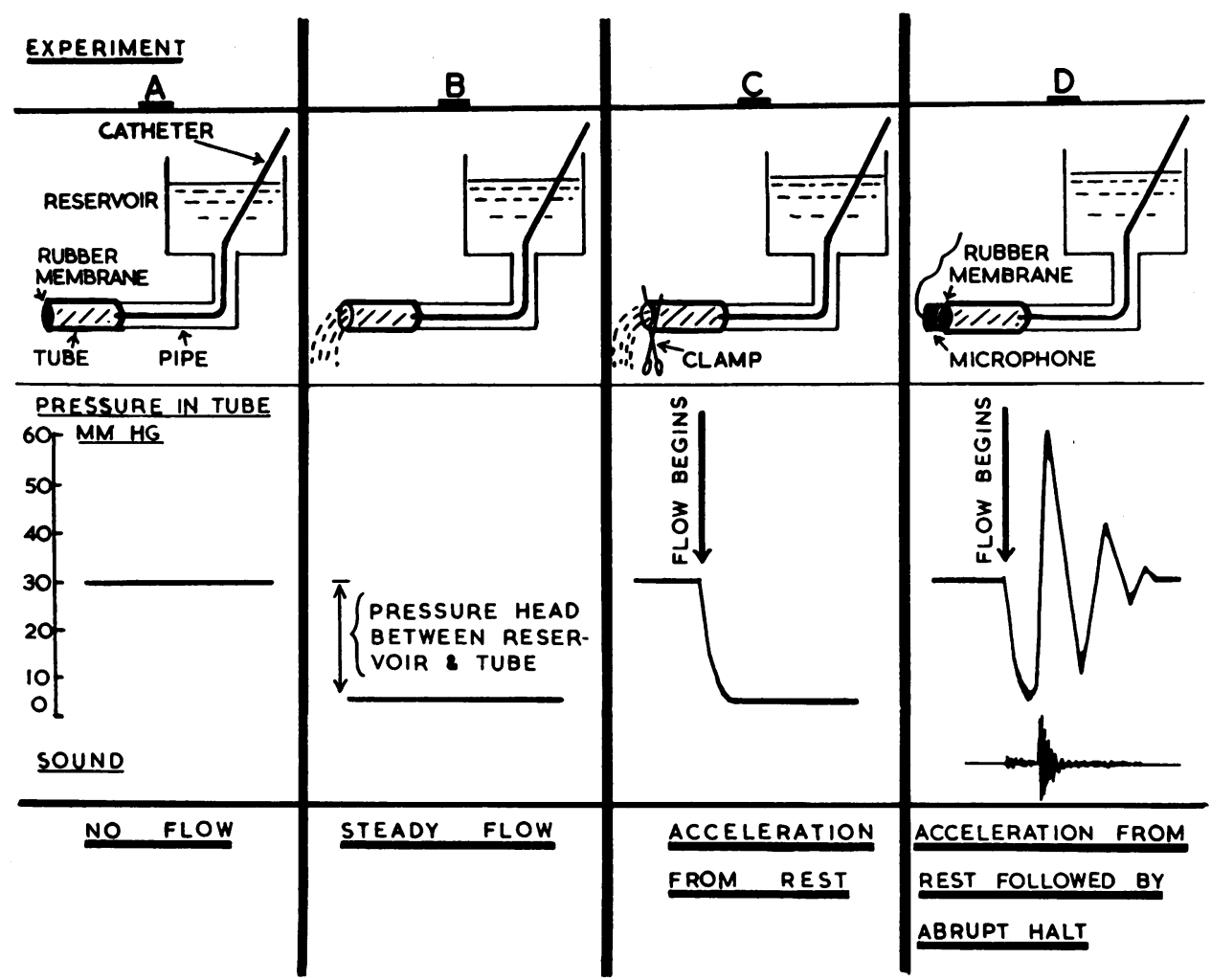

FIG. 7.-Diagram of an experiment. For explanation, see text of appendix.

In the final experiment (Fig. 7D), the end of the rubber tube was closed by the rubber membrane and the tube emptied by manual compression. The tube was then suddenly released and water flowed rapidly into it from the pipe, thus imitating ventricular filling in early diastole. Initially the same pressure changes were recorded in the tube, as when the water had accelerated from rest. Next when the closed tube had been entirely re-expanded by the water flowing into it from the pipe, there was an abrupt rise of pressure in the tube, followed by coarse oscillations, and a large sound was recorded at the beginning of this steep rise in pressure (Fig. 7D and 8). Finally, since flow had now ceased, the pressure in the tube returned to the level of pressure in the reservoir, which itself had remained unchanged throughout the experiment.

In this way, a pressure dip, followed by coarse oscillations was recorded, which was similar in broad essentials to the early diastolic dip and subsequent oscillations in the right ventricle in constrictive pericarditis. The sound produced in the experiment resembled the early diastolic sound in constrictive pericarditis in that both were coincident with the beginning of a steep rise in pressure terminating a pressure dip, which marked sudden arrest of rapid filling. In addition, the unchanged level of pressure in the reservoir which considerably exceeded the pressure in the tube during the pressure dip was comparable to the higher, relatively steady pressure in the basilic vein at the moment of the early diastolic dip in the right ventricle in constrictive pericarditis.

Conclusion. In a simple working model, in which water, under pressure, in a pipe fed from a reservoir, was allowed suddenly to flow into a small closed chamber, a sound, pressure variations and pressure differences between tube and reservoir were produced which resembled in broad essentials the early diastolic sound, the early diastolic pressure dip and the pressure difference between right heart and peripheral veins in constrictive pericarditis. 


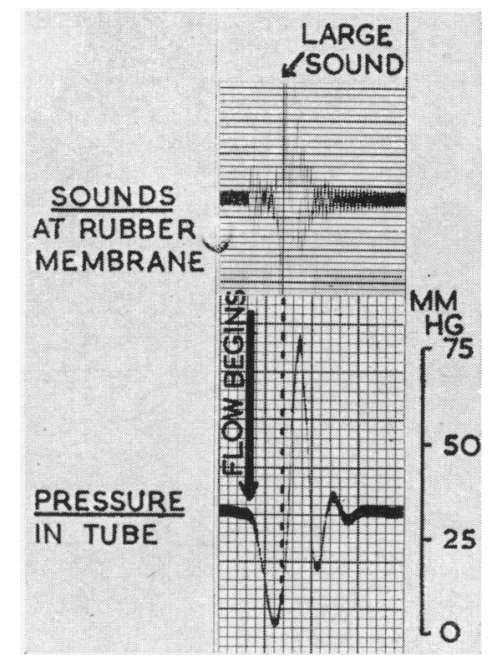

FIG. 8.-Simultaneous sound and pressure records obtained in Experiment $\mathrm{D}$. The large sound is seen to coincide with the beginning of the steep rise in pressure.

I should like to thank Dr. William Evans for his help and criticism in the preparation of this paper and Dr. Wallace Brigden, Mr. Vernon Thompson, and Mr. Geoffrey Flavell for their advice.

I also wish to thank Mr. William Dicks, Mr. Robert Burns, and Mr. Albert Gallup for their technical help.

\section{REFERENCES}

Bloomfield, R. A., Lauson, H. D., Cournand, A., Breed, E. S., and Richards, D. W. Jr. (1946). J. Clin. Invest., $25,639$.

Eliasch, H., Lagerlöf, H., and Werkö, L. (1950). Nordisk Med., 44, 1128.

Evans, W., and Jackson. F. (1952). Brit. Heart J., 14, 53.

(1954). Cardiography. Butterworths, London.

Green, H. D. (1943). Medical Physics, edited by Glasser, O., The Year Book Publishers, Chicago, 210.

Hansen, A. T., Eskildsen, P., Götzsche, H. (1951). Circulation, 3, 881.

Leatham, A. (1949). Post. Grad. med. J., 25, 568.

Lian, C., Marchal, M., and Pautrat, J. (1933). Bull. Mém. Soc. méd. Hôp Paris, 49, 20.

McKusick, V. A. (1952). Bull. Johns Hopkins Hosp., 90, 3.

Mounsey, J. P. D. (1953). Brit. Heart J., 15, 135.

Potain, C. (1856). Bull. Soc. anatom. de Paris, Aug. 29, 1856.

Wood, E. H., Geraci, J. E., Pollack, A. A., Groom, D., Taylor, B. E., Pender, J. W., and Pugh, D. G. (1948). Proc. Staff Meet. Mayo Clin., 23, 494.

Wood, P. H. (1950). Diseases of the Heart and Circulation. Eyre and Spottiswoode, London. 